\title{
Quinze ans de " petit séminaire »
}

Une analyse rétrospective

Fifteen years of the "Petit séminaire" - A Retrospective analysis

Juliette Pommier, Maxime Decommer et Véronique Patteeuw

\section{(2) OpenEdition}

Journals

Édition électronique

URL : http://journals.openedition.org/crau/533

DOI : $10.4000 /$ crau. 533

ISSN : 2547-5746

Éditeur

Éditions du patrimoine

Édition imprimée

Date de publication : 1 novembre 2012

Pagination : 31-39

ISBN : 978-2-7577-0108-9

ISSN : 1296-4077

Référence électronique

Juliette Pommier, Maxime Decommer et Véronique Patteeuw, "Quinze ans de « petit séminaire » », Les Cahiers de la recherche architecturale et urbaine [En ligne], 26/27 | 2012, mis en ligne le 01 novembre 2017, consulté le 03 mai 2019. URL : http://journals.openedition.org/crau/533 ; DOI : 10.4000/ crau.533 
Étroitement associée à la consolidation des institutions de la recherche architecturale française, I'histoire du « petit séminaire » qui rassemble les doctorants architectes à partir de 1997 touche à la fois l'histoire des lieux, celle des acteurs et des idées, celle du champ de la recherche et celle des écoles d'architecture. Véritable complément de formation, il est à la source de certaines affiliations scientifiques et professionnelles, esquissant un réseau à travers la France, des laboratoires aux écoles d'architecture en passant par les revues. À mi-chemin entre la réflexion scientifique et le soutien psychologique, entre l'échange d'informations sur le monde de la recherche et le partage des références culturelles, génération après génération, le " petit séminaire » a produit plusieurs sortes d'effets.

\section{Quinze ans de « petit séminaire "}

\section{Une analyse rétrospective}

JULIETTE POMMIER

MAXIME DECOMMER

VÉRONIQUE PATTEEUW

Baptisé par opposition au séminaire des " grands " - celui des enseignants-chercheurs et des directeurs de thèse -, le "petit séminaire » est né au détour d'un couloir, dans les locaux du DEA inter-écoles d'architecture "Le projet architectural et urbain », au milieu des années 1990'. Dès ce moment, il se définit comme une réunion périodique de doctorants organisée sans leurs encadrants dans le but de discuter de leurs recherches, de leurs problématiques et de leurs méthodes, mais aussi plus généralement des différents aspects de la vie du thésard. À mi-chemin entre la réflexion scientifique et le soutien psychologique, entre l'échange d'informations sur le monde de la recherche et le partage des références culturelles, cette rencontre régulière, génération après génération, a produit plusieurs sortes d'effets.

Au-delà de l'entraînement à l'expression orale et à l'argumentation, ce véritable complément de formation est à la source de certaines affiliations scientifiques et professionnelles, esquissant un réseau à travers la France, des laboratoires aux écoles d'architecture en passant

1. Fondé à la rentrée 1991-1992, ce diplôme d'études approfondies (DEA) affilié à l'université Paris vIII constituait l'année de préparation au doctorat, prémisse nécessaire à l'inscription dans le champ Urbanisme, puis Architecture, à Paris VIII. Suite à l'institutionnalisation progressive du doctorat en architecture, de son intégration dans des écoles doctorales (2000) à la réforme LIMADO (mise en place en 2005-2006), le DEA a disparu. Il est aujourd'hui conseillé aux étudiants souhaitant s'inscrire en doctorat de suivre un master recherche dans les écoles nationales supérieures d'architecture. 
par les revues. Aux effets épistémologiques et sociaux se sont ajoutés des fruits plus tangibles : des collaborations au sein d'appels d'offre de recherche; des contributions à des revues spécialisées ${ }^{2}$; l'organisation de cycles de séances de séminaire, de colloques et de publications ${ }^{3}$.

Chacune de ces activités participe d'une histoire à plusieurs niveaux de lecture : sur 15 ans, trois générations de doctorants animent des cycles de trois à cinq ans, selon leurs trajectoires personnelles, leurs disponibilités et leurs ressources - en fonction des structures pédagogique et administrative du doctorat. Étroitement associée à la consolidation des institutions de la recherche architecturale française, I'histoire du petit séminaire touche à la fois celle des lieux, des acteurs et des idées, celle du champ de la recherche et celle des écoles d'architecture.

Après une première tentative avortée en 1995, le petit séminaire est fondé en 1997 par les doctorants du laboratoire Acs à l'école d'architecture de Paris-Villemin. Organisées dans les locaux du laboratoire, les séances associent l'exposé et le débat autour de la recherche de l'un des doctorants. C'est une discussion à bâtons rompus sur les difficultés méthodologiques, qui s'élargit progressivement à d'autres modes d'échange, de la relecture d'articles à l'élaboration de réponses aux appels d'offre de recherche, en passant par le partage des vacations d'enseignement et des réseaux professionnels. Ouvert à tous les doctorants en architecture, le séminaire repose de fait sur la présence et l'animation des allocataires de recherche du laboratoire ACS ${ }^{4}$.

Quatre ans plus tard, la deuxième génération entre en scène, au moment où la précédente amorce son intégration dans les milieux professionnels et dans les écoles d'architecture ${ }^{5}$. La génération 2001 retourne symboliquement et spatialement aux sources : les séances ont lieu dans les locaux du DEA, à l'école de Paris-Belleville, et s'appuient davantage sur la collaboration entre les doctorants des deux laboratoires ACS et IPRAUS ${ }^{6}$.

Après une rupture temporelle et institutionnelle, la troisième génération se constitue dans un contexte très différent : le DEA a disparu en 2005 et le doctorat, à présent encadré par les écoles d'architecture, manque à ses débuts de visibilité. De nouveaux doctorants inscrits à l'école de Paris-Malaquais dans les laboratoires Acs et $\operatorname{LIAT}^{7}$ refondent le petit séminaire en 2008.

Et bientôt, 15 ans. Que reste-t-il du cycle rapide des promotions qui se succèdent, se rencontrent, s'entendent ou s'ignorent? Étonnamment, beaucoup de choses. Au delà de l'entraide et de l'initiation à la recherche, il semble qu'il se joue dans ces années-là des effets d'identification et d'affiliation, de la construction de réseaux à la structuration des cultures scientifiques, jusqu'à la constitution de visions du métier de chercheur, et du rôle qu'il peut jouer dans le champ architectural.

Dans cet article, nous proposons une analyse rétrospective de l'expérience de ces 15 ans; une lecture du petit séminaire dans son épaisseur, au-delà des ruptures et continuités historiques. L'analyse s'effectuera sur la base des témoignages de plusieurs protagonistes de cette histoire ${ }^{8}$, en articulant les modes de constitution du petit séminaire, les sociabilités particulières qui en découlent, ainsi que les cultures qui s'y développent. En examinant les conditions d'existence du petit séminaire, nous croiserons les approches pédagogique, sociologique et épistémologique.
2. La revue Labyrinthe puis In Extenso, Le Visiteur, Criticat.

3. "Histoires d'histoire de l'architecture », séminaire thématique sur l'historiographie de l'architecture et de la ville en France, organisé en 2000-2001, à l'initiative des membres du petit séminaire (génération 1997); « Autour de l'étrangeté. Une rencontre entre architecture et politique », colloque tenu en juin 2010, à l'initiative des membres du petit séminaire (génération 2008), associant l'Énsa de
Paris-Malaquais et Sciences-Po, publication en cours.

4. Laboratoire ACS, Architecture, Culture, Société, $x\left|x^{e}-x\right|^{e}$ siècles, Énsa de Paris-Malaquais (ancienne Énsa de Paris-Villemin) a été fondé en 1990, et il est dirigé par Monique Eleb. Depuis le $1^{\text {er }}$ janvier 2010, le laboratoire Acs est rattaché à l'UMR AUSSER 3329. Site de I'UMR: http://www.umrausser.cnrs.fr/
5. Même si les thèses de doctorat de la génération 1997 ne seront soutenues pour la plupart qu'au milieu des années 2000, la réalité des allocations finissantes et des opportunités professionnelles naissantes contraint les membres du petit séminaire à se consacrer à d'autres activités.

6. L'IPRAUS, Institut Parisien de Recherche Architecture Urbanistique, Énsa de Paris-Belleville a été fondé en 1986, et il est dirigé par Jean-Philippe Garric. Depuis 


\section{Apprendre à faire de la recherche Le petit séminaire comme instrument pédagogique}

Dans le milieu académique, le doctorant a un statut ambigu auquel il doit souvent seul donner un sens. II est nécessaire qu'il invente son propre parcours, qu'il s'agisse de mener à terme la thèse, d'accéder à l'enseignement ou à la recherche, ou encore de valoriser ses propres travaux. À cette position singulière fait écho le cadre institutionnel complexe du doctorat en architecture. Dans le foisonnement des différentes structures (le PRES, pôle de recherche et d'enseignement supérieur, l'école doctorale, l'école nationale supérieure d'architecture, le laboratoire), c'est souvent ce dernier qui favorise la cohésion des doctorants. Mais à l'instar de la thèse, s'organisant suivant des temporalités multiples et irrégulières, la vie du laboratoire connaît des périodes d'intense activité ou de délaissement relatif. Dans ce contexte, le petit séminaire constitue une alternative : aussi bien en termes d'échange et d'encadrement que de gestion du temps et de l'espace de la recherche, il participe à la structuration du cadre de vie du doctorant.

\section{Critique mutuelle et entraide}

L'activité structurelle du petit séminaire réside dans la critique réciproque et dans la solidarité entre doctorants. Réunis autour d'une présentation mensuelle devant leurs pairs, les participants partagent leurs états d'avancement, leurs connaissances et leurs méthodes, leurs difficultés et leurs questions. C'est un lieu de confiance, qui permet de se délester de la pression de la hiérarchie, du jugement et de la concurrence : on y est tous chercheurs.
Suivant de près les phases de la thèse, le petit séminaire peut se moduler selon le parcours et les besoins de ses membres. Des rapports d'entraide différents s'y développent : échange de références et de sources lors de la phase d'exploration du champ (par exemple au travers de participations communes à des cours et séminaires, au sein de l'école doctorale et ailleurs, en particulier à l'École des hautes études en sciences sociales, EHESS et à l'Institut national d'histoire de l'art, INHA); critique et réflexion aux moments de reproblématisation et d'analyse; ou encore temps de concentration et de relecture critique lors de l'écriture.

Chaque séance offre au doctorant un moment d'évaluation scientifique et de situation par rapport à la communauté. Cette opportunité d'échange dépend de l'équilibre intergénérationnel. L'histoire du petit séminaire témoigne de la difficulté à le conserver : quand d'autres objectifs prennent le pas sur l'avancement de la thèse, ou quand des rapports hiérarchiques se dessinent, les tensions remplacent la confiance et le séminaire ne joue plus son rôle. L'équilibre du séminaire repose donc sur l'équilibre entre les participants.

\section{Profils}

Ces participants, au cours des trois générations, présentent des similarités d'ordre multiple déterminées en grande partie par leurs moyens d'existence : les doctorants recevant une allocation de recherche ou une bourse sont toujours les initiateurs de la création ou de la reprise du projet ${ }^{9}$. Bénéficiant souvent d'une place de travail dans un laboratoire et d'un statut reconnu par les chercheurs confirmés, ils disposent de plus de temps le $1^{\text {er }}$ janvier 2010, le laboratoire Acs est rattaché à I'UMR AUSSER 3329.

7. Issu du laboratoire GRAl (Groupe de recherche sur l'architecture et les infrastructures) créé en 1990 à l'Énsa de Versailles, le laboratoire LIAT, Infrastructure, Architecture, Territoire, a rejoint l'Énsa Paris-Malaquais en 2008, et il est dirigé par Dominique Rouillard.
8. Pierre Bourlier (entretien le 23 février 2011) ; Pierre Chabard (entretien le 24 février 2011) ; Anne Debarre (entretien le 23 février 2011) : Valérie Lebois (entretien le 23 février 2011); Pauline Lefort (entretien le 25 février 2011): Joëlle Trouvé (entretien le 22 février 2011).
9. L'allocation de recherche est de 1374 euros net par mois; elle est attribuée pour une période de 3 ans. L'allocation d'études spécialisées du ministère de la Culture et de la Communication est de 900 euros net par mois; elle est accordée pour une année et soumise au renouvellement deux fois. 
pour s'investir dans diverses activités leur permettant d'acquérir de l'expérience et des compétences. Certains sont représentants des doctorants dans les instances des écoles, d'autres ont accès au monitorat ou à des vacations d'enseignement. Ces activités et statuts développent chez ces doctorants un sentiment de responsabilité. Percevant leur allocation comme un tribut à rendre au laboratoire, ils s'investissent dans des missions de valorisation ${ }^{10}$, et mettent à disposition des doctorants leurs compétences et leur temps, notamment dans l'organisation du petit séminaire. La correspondance entre durée de l'allocation et cycle d'existence de chacun des petits séminaires confirme ce lien entre financement et implication.

\section{L'importance du cadre}

À chaque (re)création, I'autogestion semble jouer un rôle important dans le développement des différentes formes du petit séminaire. Il demeure souvent ignoré des directeurs de thèse pendant les premières semaines, voire les premiers mois, de fonctionnement et conserve par la suite une forme presque secrète : réponse du doctorant à son statut ambigu, moyen de se détacher temporairement de ses enseignants ou encore prétention à une autonomie totale? La discrétion autour du petit séminaire est avant tout un moyen de protéger son statut à part, à la marge des institutions; un espace qui ne cherche pas à être visible, autorisant l'expérimentation.

Si le petit séminaire naît du désir d'échanges, la proximité spatiale s'avère déterminante. Chaque génération connaît (et choisit) son propre lieu : une salle de cours rue Frémicourt, une autre salle de cours dans les locaux du DEA à Belleville, puis un centre de documentation à Paris-Malaquais.

Cette opportunité d'échanges offerte aux doctorants par les doctorants n'intéresse pourtant pas tout le monde. Obligés de s'assurer d'un revenu, beaucoup d'étudiants n'ont tout simplement pas le temps de s'y investir; d'autres sont bien entourés dans leurs laboratoires et ne ressentent pas le besoin de l'échange; enfin, $d$ 'autres encore conçoivent la thèse comme un travail personnel, en relation exclusive avec le directeur de thèse. Même si le petit séminaire est pensé comme un lieu ouvert, il se limite souvent à ceux dont les sujets de thèse ou les cultures scientifiques présentent des similitudes. La proximité physique est importante pour la constitution du groupe, mais les affinités de personnes, de sujets et de cultures le sont davantage.

Le petit séminaire répond donc en premier lieu à un besoin d'échanges et d'encadrement. Très différent de I'encadrement du directeur de thèse, il constitue un lieu d'auto-éducation, un complément d'une nature multiple portant non seulement sur les objets et les méthodes de recherche mais aussi sur la compréhension du milieu, la gestion du temps long de la thèse et plus globalement sur la vie du thésard ${ }^{11}$.

\section{Entrer dans le champ de la recherche architecturale Une initiation au milieu}

À chaque génération, plusieurs modèles ont déterminé la forme particulière du petit séminaire. Procédant par emprunt, par opposition ou par interprétation de ces
10. Rédaction du journal du laboratoire, présentation de la recherche lors des Journées «portes ouvertes » des écoles...
11. Plusieurs écoles d'architecture, notamment dans les pays anglo-saxons, organisent le doctorat en étapes. Celles-ci offrent au doctorant des jalons tout au long du parcours de la thèse et facilitent ainsi la maîtrise de la temporalité de la recherche. 
modèles, chaque groupe n'a eu de cesse de fabriquer son propre petit séminaire. Les doctorants ont souvent rencontré ces modèles au cours de leurs études, dans les écoles d'architecture ou à l'université ${ }^{12}$, au travers de séminaires donnés en master ou pendant le DEA. Des références à des systèmes plus anciens, pourtant de moins en moins appliqués dans les écoles d'architecture, sont aussi présentes : c'est le cas de l'Atelier des Beaux-Arts, système qui par son organisation verticale développait entraide et soutien entre aînés et plus jeunes. Des modèles "non-académiques " apparaissent également : le comité de lecture, la discussion informelle et parfois la psychothérapie de groupe... Enfin, de manière moins consciente, un modèle plus diffus s'est affirmé à chaque génération : celui du laboratoire de recherche ou, plus exactement, de la représentation qu'un doctorant peut s'en faire lorsqu'il est en thèse. Chacune de ces formes, construite sur des regroupements sociaux différents et associée à des activités diversifiées, révèle ce qui se joue pour les membres du petit séminaire autant à l'intérieur de la structure qu'à l'extérieur de celle-ci, dans le milieu de la recherche architecturale.

\section{Identification et appartenance}

Le petit séminaire, à chaque génération, c'est avant tout la naissance d'un groupe. La résonance des sujets de thèse et le partage d'approches, de références et de méthodes assurent la cohésion de celui-ci, et l'affirmation par des actes et des comportements singuliers lui permet d'établir sa propre identité. À chaque génération, c'est quasiment la recréation d'un mythe fondateur que l'on observe. Pour les acteurs de la génération 1997, le regroupement est évidemment inédit, mais la forme secrète qui est assignée au petit séminaire concourt à la conservation d'une image mythique. Pour la génération 2001, c'est une définition par l'opposition qui est flagrante, autant dans les approches, les contenus que les filiations. Même si la forme est reprise de la génération précédente, le traitement est complètement différent, la démarcation visible et affirmée. Enfin, pour la génération 2008, c'est l'autonomie qui est mise en avant, et la ressuscitation d'un projet laissé pour mort quelques années auparavant. Ces processus, impalpables dans le temps de l'action sont à posteriori racontés comme tels par les acteurs et contribuent à leur identification comme groupe ou, plus précisément, comme génération ${ }^{13}$.

\section{Faire ses preuves. Les autres activités du petit séminaire}

La différenciation entre les générations est aussi induite par la spécificité des activités que chacune a choisi de développer. Au delà des activités structurelles, chaque génération conçoit pour le petit séminaire d'autres objectifs, empruntant à nouveau à des modèles identifiables dans le champ de la recherche architecturale. Ces activités ont souvent pour point commun la volonté d'aboutir à une production concrète, perçue comme moyen supplémentaire d'affirmer une identité propre.

La génération 1997 utilise parfois les séances du petit séminaire pour examiner les appels d'offres de recherche, pour en comprendre les contenus et les attentes et pour élaborer des réponses, de manière individuelle ou collective. De plus, cette génération
12. Parmi les modèles universitaires figurent les séminaires donnés par Hubert Damisch ou Christian Topalov à l'École des hautes études en sciences sociales, EHESS.
13. C'est notamment sous cet angle que Jean-Louis Violeau explore « la génération 68 » dans Les architectes et Mai 1968, Paris, Éditions Recherches coll. «Focales », 2005. 
organise le cycle de conférences Histoires d'histoire de l'architecture, en parallèle des séances habituelles du séminaire ${ }^{14}$.

La génération 2001 investit le modèle du séminaire et le développe de manière plus poussée : elle initie des séances méthodologiques accordant une place prépondérante à la sociohistoire, en s'inspirant du séminaire de doctorants de Christian Topalov à l'EHESS. Le modèle du comité de lecture est aussi emprunté pour la production d'articles qui seront publiés dans la revue de sciences sociales Labyrinthe ${ }^{15}$. Peu à peu, le modèle développé pour des activités doctorales est transposé pour des activités professionnelles : les oraux blancs de concours pour l'obtention des postes d'enseignants complètent ceux des soutenances de thèse.

Pour la génération 2008, le petit séminaire sert d'abord de lieu d'échange méthodologique mais la construction théorique collective n'est pas au rendez-vous. Le trop grand nombre de participants et les grandes divergences culturelles et personnelles expliquent cet échec. L'année d'après, un petit groupe issu de cette génération s'extrait pour porter un nouveau projet concret : un colloque de doctorants, accueillant aussi des chercheurs confirmés; la formation par les aînés, absente l'année précédente, réapparaît. II est à noter que la production concrète est facilitée par le parcours personnel de certains des protagonistes, important chacun leur expérience professionnelle au sein du petit séminaire.

La caractéristique commune à chacune des générations est de pouvoir expérimenter, au sein de cette structure non institutionnelle et entre pairs, les activités d'un véritable laboratoire de recherche. Le petit séminaire apparaît ainsi également comme le lieu de la simulation de la vie de laboratoire, le lieu où par leurs actes et le développement de leurs positions scientifiques, les doctorants prennent progressivement conscience du statut, de l'être et du devenir du chercheur.

\section{Devenir chercheur \\ Une construction épistémologique}

La prise de conscience de la valeur du statut de chercheur provoque l'acquisition d'une certaine culture scientifique de la part du doctorant, et vice versa. Dans cette interaction entre l'apprentissage du métier de chercheur et l'acquisition, voire la production, des savoirs et savoir-faire nécessaires à la recherche, les dimensions pédagogique, sociale et épistémologique de la thèse s'entrecroisent - et s'amplifient au sein du petit séminaire. Dans ce tissage complexe, identification sociale au groupe résonne avec affiliation scientifique, générant de manière plus ou moins consciente des familles épistémologiques caractérisées.

\section{Culture scientifique et affiliations croisées}

De 1991 à 2005, le DEA a agi comme lieu d'affiliation à la fois sociale et scientifique ${ }^{16}$. Au travers des cours, de l'encadrement des mémoires et des stages en laboratoire, les enseignants ${ }^{17}$ ont transmis non seulement leurs concepts et leurs méthodes, mais aussi une certaine vision du champ de la recherche architecturale, structuré autour de problématiques spécifiques, telles que la généalogie de l'habitat, les 
transferts culturels, I'historiographie de l'architecture ou I'analyse des mécanismes de fabrication urbaine.

L'affiliation au petit séminaire se superpose à celle dU DEA : elle la spécialise, la transpose, et parfois la contredit. Celui-ci constitue souvent le langage commun qui permettra plus tard le rapprochement des doctorants au sein du petit séminaire : c'est parce qu'ils se comprennent qu'ils peuvent travailler ensemble, tout en s'enrichissant des différences culturelles au sein du groupe - le revers de cette cohésion est évidemment l'exclusion des " autres", et en particulier des non-architectes, qui restent minoritaires. D'autres affiliations plus anciennes viennent renforcer cette résonance entre les idées et les sujets : la génération 1997 est plutôt issue de l'école d'architecture Paris-Villemin, celle de 2001 provient majoritairement de Paris-Belleville, et celle de 2008 plutôt de ParisMalaquais. En somme, les affiliations développées au sein du petit séminaire proviennent à la fois du DEA et des écoles d'architecture, produisant dès lors une similarité culturelle. Mais à y regarder de plus près, les choses sont plus complexes.

Par exemple, si l'on réexamine la forte orientation sociohistorique ${ }^{18}$ qui caractérise la génération 2001, une convergence de faits et de rencontres marque la naissance et l'amplification du phénomène : d'une part la découverte du séminaire de Christian Topalov, d'autre part la présence d'une doctorante psychosociologue dans cette génération, et enfin la critique d'une histoire des "influences " que seule une sociohistoire des acteurs et des objets semble permettre d'éviter.
De la même manière, la génération 1997 s'est davantage interrogée sur l'historiographie grâce à la résonance entre les approches des doctorants en présence, qui découvraient alors une vision critique de l'histoire. Elle se caractérise aussi par sa source : les séminaires d'Hubert Damisch, où I'on expose " la cuisine interne » de la recherche. À l'opposé d'un cours magistral, au sein de ces séminaires, étudiants et enseignants quittent pour un temps leurs positions et leurs rôles respectifs pour partager à armes égales leurs doutes, leurs angoisses et leurs méthodes. Peut-on en déduire que l'identité épistémologique du groupe se construit au travers d'une posture critique?

\section{L'autonomie scientifique}

Le cas de la génération 2008 est un peu différent. D'abord, parce que le DEA n'est plus, et qu'aucune cohésion de promotion ne préexistait au petit séminaire. Ensuite, parce que les inscriptions en doctorat se font au sortir du master, quasiment sans spécialisation préalable à la recherche, et que les sujets de doctorat sont par conséquent moins construits. De fait, le rapprochement des doctorants passe d'abord par le partage du lieu. Dans ce contexte, qu'en est-il d'une affiliation épistémologique?

L'examen des activités de cette génération révèle plusieurs aspects de l'apprentissage de la recherche et de l'émergence d'une conscience des enjeux épistémologiques. En premier lieu, la transmission intergénérationnelle agit comme catalyseur dans le processus de prise de conscience, d'appropriation et de reconstruction pour soi des méthodes de la recherche 


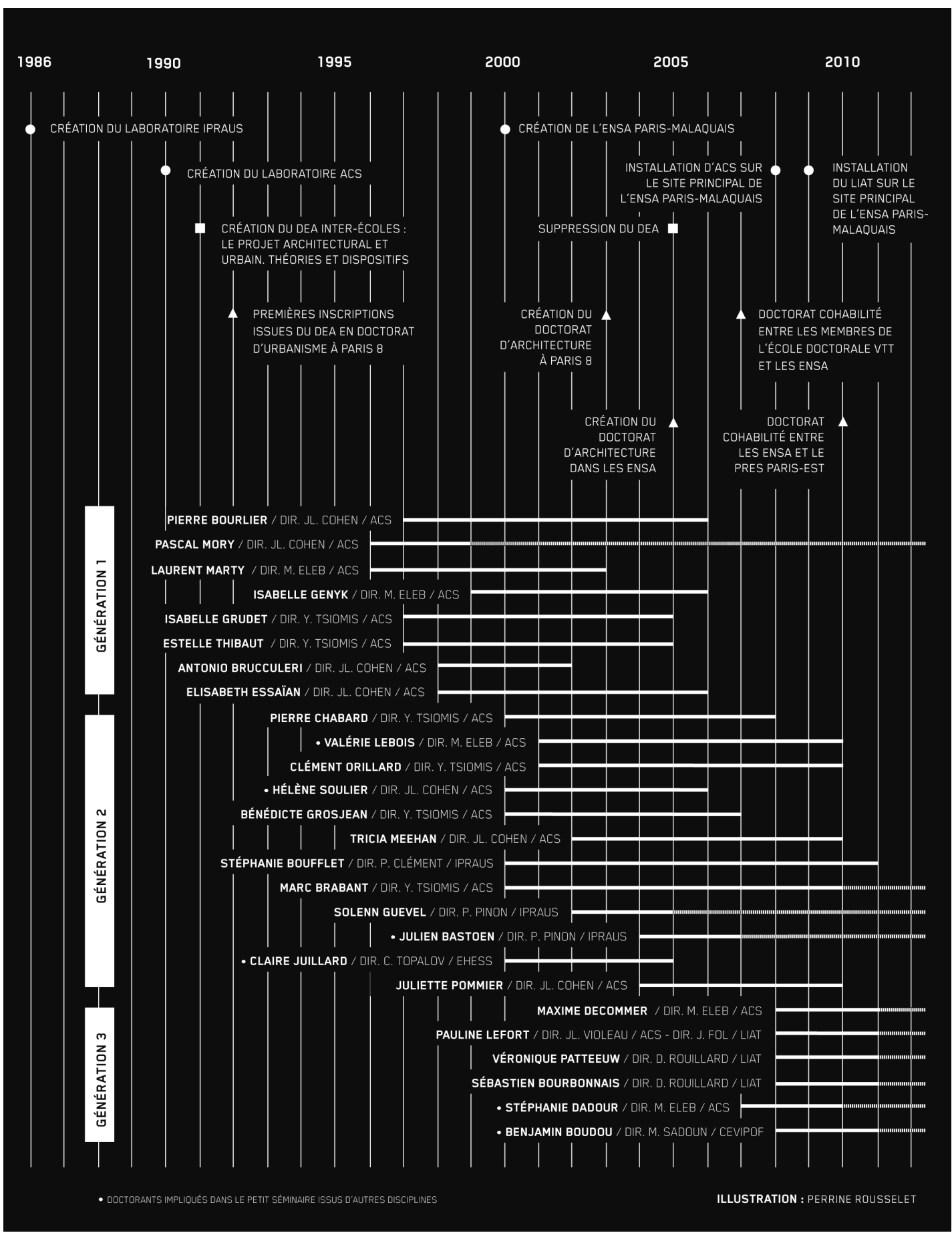

Chronologie du petit séminaire

(C) Illustration Perrine Rousselet 
architecturale. Non seulement les postures scientifiques des aînés sont constituées et transmissibles, mais en plus la critique mutuelle qui fonde le petit séminaire est avant tout une critique des aînés. En second lieu, elle démontre l'utilité d'une relative autonomie - mais non d'un isolement - pour construire une position scientifique : c'est notamment au travers de l'organisation d'un colloque que les doctorants ont pu expérimenter l'interdisciplinarité, la classification des approches et des objets en différents ateliers, et l'interaction fructueuse avec des chercheurs plus chevronnés, qui sont intervenus en tant que discutants.

Ainsi, l'«apprentissage de la recherche par la recherche ${ }^{19}$ " ne passe pas uniquement par l'insertion du doctorant dans les recherches du laboratoire - ce qui constitue cependant une opportunité fructueuse d'apprentissage, ne nous méprenons pas - mais aussi par le montage de ses propres projets, voire par ses erreurs.

\section{Conclusion}

Au prisme d'une analyse rétrospective, le petit séminaire se révèle un complément important à l'accompagnement du doctorant, tout au long de son parcours. Cette structure non institutionnelle et non hiérarchique a pris forme dans l'autocritique et dans l'entraide et a contribué à la compréhension des outils et des méthodes du chercheur ainsi qu'à celle du milieu et des cultures scientifiques. Le petit séminaire concourt aussi à la formation du doctorant aux temps de la recherche, à la conscience de sa propre pensée en construction, et à son devenir de chercheur. Ce véritable laboratoire permet également de saisir la recherche comme une dynamique continue et collective, et non comme une découverte solitaire.

La construction d'une culture scientifique, étape obligée du parcours du doctorant, peut se lire comme une aventure complexe, faite de rencontres, d'épreuves et d'expériences diversifiées, qui n'est pas sans rappeler l'adage selon lequel le chemin de la recherche est plus important que le résultat. Sur ce chemin, le petit séminaire joue à la fois le rôle de la source et du compagnon de route. II aide à se situer dans l'espace et dans le temps du champ de la recherche architecturale, et à s'en faire progressivement une représentation plus précise. Le jeune chercheur est ainsi confronté dès ces premiers pas aux multiples dimensions de la recherche et du milieu : l'apprentissage (perpétuel) de la recherche, le champ scientifique en mouvement constant, la construction (sociale) des idées et des postures du chercheur.

L'histoire du petit séminaire met en lumière ses modes de constitution et ses conditions d'existence ainsi que les familles et cultures qui en découlent. Mais cette histoire et les témoignages de ses protagonistes permettent surtout de rendre compte de la dimension quotidienne de la vie du thésard et la fonction que le petit séminaire y assure. Dans une vision prospective, cette analyse propose de contribuer à la réflexion sur les structures du doctorat, en intégrant, à l'organisation du cursus, les questions de gestion du temps, d'initiation au milieu, d'identification et d'appartenance sociales, de culture et d'autonomie scientifiques.

19. Voir à ce sujet l'analyse de Clément Orillard, ancien membre organisateur du petit séminaire, "Point de vue d'un architecte doctorant », 17 juin 2004. Consultable en ligne : http://slr39.free.fr/ article.php3?id_article=20. 